\title{
FDTD Analysis of a Gigahertz TEM Cell for Ultra-wideband Pulse Exposure Studies of Biological Specimens
}

\author{
Zhen Ji*, Student Member, IEEE, Susan C. Hagness, Senior Member, IEEE, John H. Booske, Senior Member, IEEE, \\ Satnam Mathur, Member, IEEE, and Martin L. Meltz, Member, IEEE
}

\begin{abstract}
Gigahertz transverse electromagnetic (GTEM) transmission cells have been previously used to experimentally study exposure of biological cells to ultra-wideband (UWB), monopolar, electromagnetic pulses. Using finite-difference time-domain (FDTD) simulations we examine the time-dependent electric field waveforms and energy dose spatial distributions within a finite volume of biological cell culture medium during these experiments. The simulations show that when one or more flasks containing cell culture media are placed inside the GTEM cell, the uniform fields of the empty GTEM cell are significantly perturbed. The fields inside the cell culture medium, representing the fields to which the biological cells are exposed, are no longer monopolar and are spatially highly nonuniform. These effects result from a combination of refraction and distortion of the incident wave, combined with excitation of resonant eigenmodes within the cell culture medium volume. The simulations show that these distortions of the incident waveform may be mitigated by supporting the sample on a high permittivity pedestal and modifying the incident waveform to more closely approximate a Gaussian pulse. Under all simulated conditions, the estimated maximum temperature rises are completely negligible, ensuring that any experimentally observed unusual cell function or histopathology can be associated with nonthermal effects.
\end{abstract}

Index Terms-Dosimetry, FDTD methods, gigahertz transverse electromagnetic (GTEM) cell, ultra-wideband (UWB) exposure.

\section{INTRODUCTION}

$\mathbf{T}$ HE increased use of ultra-wideband (UWB) radar technology in military and civilian applications has raised questions about potential biological effects, thereby motivating a number of recent UWB electromagnetic pulse (EMP) exposure studies [1]-[11]. A commonly used protocol for

Manuscript received March 22, 2005; revised September 29, 2005. This work was supported in part by the Air Force Office of Scientific Research under Grant F49620-01-1-0349 through a subcontract from UTHSCSA, and in part by the Department of Defense under MURI Program FY01 "RF Bioeffects" through a consortium grant with Old Dominion University and managed by AFOSR. Asterisk indicates corresponding author.

*Z. Ji is with the Department of Electrical and Computer Engineering, University of Wisconsin, 1415 Engineering Drive, Madison, WI 53706 USA (e-mail: zji@wisc.edu).

S. C. Hagness and J. H. Booske are with the Department of Electrical and Computer Engineering, University of Wisconsin, Madison, WI 53706 USA (e-mail: hagness@engr.wisc.edu; booske@engr.wisc.edu).

S. Mathur was with McKesson BioServices, USAMRD. He is now with Costal Bend College, Pleasanton, TX 78064 USA.

M. L. Meltz is with the Department of Radiation Oncology, University of Texas Health Science Center at San Antonio (UTHSCSA), San Antonio, TX 78229 USA.

Digital Object Identifier 10.1109/TBME.2005.863959 investigating the biological effects of electromagnetic wave exposure is to conduct a controlled experiment wherein living cell cultures are placed in a well-defined field environment, such as that of a transverse electromagnetic (TEM) wave, and the cellular function or histopathology is carefully analyzed. For baseband EMP exposures, a TEM wave can be generated in a closed, shielded structure known as a gigahertz TEM transmission cell (GTEM cell) [12]. The GTEM cell is a broadband variant of the Crawford TEM cell [13]. It consists of a tapered rectangular coaxial transmission line, as illustrated by the photographs and the schematic of Fig. 1. The inner conductor (the septum) is a flat tapered plate and the outer conductor is a rectangular tube with a cross-sectional area that increases in size along the line. The apex of the GTEM cell is connected to an RF pulse generator. UWB pulses propagate longitudinally along the line and are absorbed by low- and high-frequency terminations at the load end. Flasks containing biological cell cultures are placed within the GTEM cell along its bottom surface beneath the septum and are exposed to the propagating fields.

Precise knowledge of the electromagnetic dose of each biological specimen is critical in accurately establishing a biological response-electromagnetic dose relationship. The electromagnetic properties of empty GTEM cells used as electromagnetic compatibility test chambers have been characterized using analytical calculations [14], [15] numerical simulations [16], [17], [19] and experimental measurements [17], [19]. Such characterizations of field patterns in the unloaded GTEM cell cannot be used to reliably predict the spatial and temporal field variations inside cell-culture flasks inserted into the GTEM cell. The electromagnetic properties of a GTEM cell loaded with a cuvette has been investigated via numerical simulations [18]; however, that study was conducted in two-dimensional rather than three-dimensional (3-D). Experimental dosimetry techniques rely upon the use of in situ sensors, such as D-dot sensors [20], [21], placed inside the flasks. These sensors provide data at a small number of locations inside the flask and primarily sense only one vector field component. Furthermore, the sensing volume of these sensors may not be sufficiently small to capture rapid spatial and temporal field fluctuations inside the flasks. Given these experimental limitations, a full-wave time-domain computational electromagnetics technique such as the finite-difference time-domain (FDTD) method [22] is regarded as a practical means of acquiring detailed dosimetry information, as demonstrated over the past 


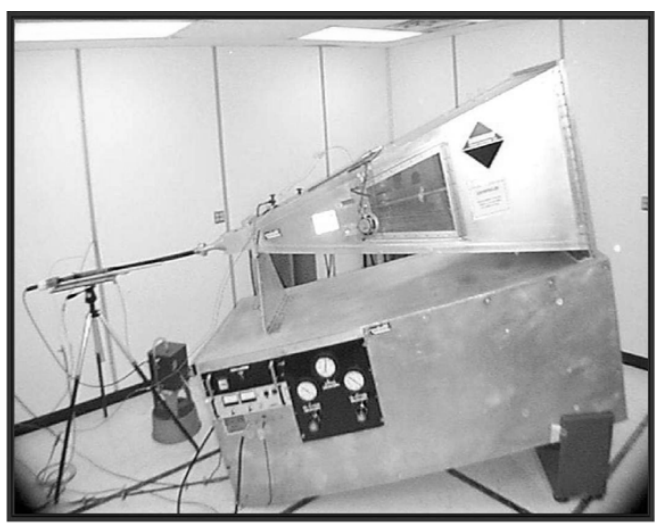

(a)

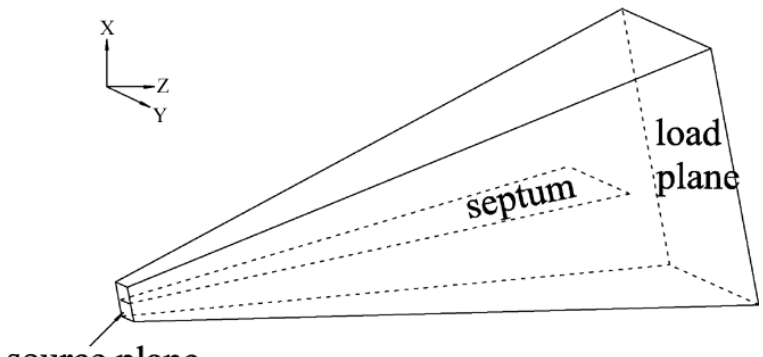

(b)

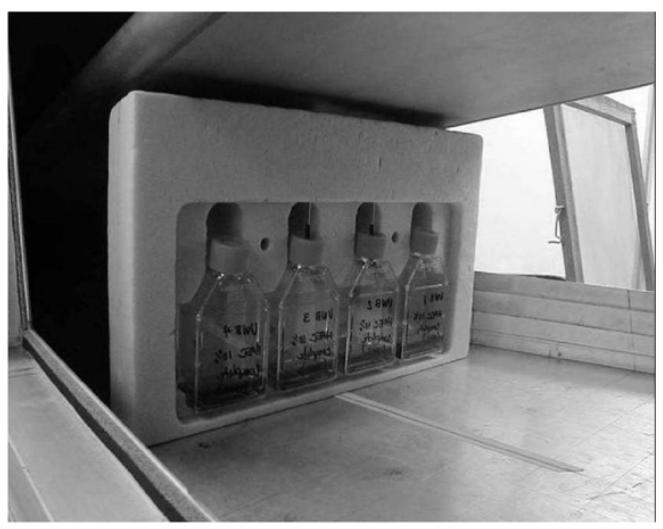

(c)

Fig. 1. (a) Photograph of an UWB exposure system comprised of a GTEM cell (on top) connected to a pulse generator (beneath). (b) Three-dimensional schematic of the GTEM cell showing the inner conductor (the septum) and the outer conductor (the flared rectangular tube). (c) Photograph of the interior of the GTEM cell loaded with four T-25 flasks.

decade by numerous FDTD analyzes of narrowband in vitro exposure systems (see, for example, [23]-[26]).

In this paper, we report a comprehensive 3-D FDTD analysis of a GTEM cell used in recent UWB EMP exposure studies conducted by the University of Texas Health Science Center in San Antonio, TX in collaboration with the US Army Medical Research Detachment at Brooks City-Base, TX. The objective of those exposure studies is to examine low-level nonthermal cellular and molecular responses occurring after exposure of mammalian cells to high average peak power EMPs with pulse widths on the order of $1 \mathrm{~ns}$. As the results of our analysis will show, the spectral content of the UWB pulse relative to the electrical size of the specimen volume strongly influences the tem- poral and spatial coupling of electromagnetic energy into the specimen. In our study, we investigated the regime where the pulse width is comparable to the pulse rise time. This complements the results of [18] which focused on a specific regime in which the pulse width is much larger than rise time.

In Section II, we describe the configuration of the GTEM cell implemented in the FDTD model and the details of the FDTD simulations and data extraction, including the calculation of the spatial distribution of the absorbed energy density (AED). In Section III, the FDTD model is validated, and the evaluations of the effect of flask walls and flask configurations are presented. Examination of unique waveform reverberation effects inside the cell culture medium is presented in Section IV. Dosimetry calculations for a cell culture medium flask are included in Section V. In Section VI, electric field polarity changes in the culture medium are described. A summary of the findings is presented in Section VII.

\section{FDTD MODEL OF THE GTEM CELL}

The configuration of the GTEM cell implemented in the FDTD model corresponds to the UWB exposure system shown in Fig. 1(a), designed and built by Sandia National Laboratories and located in a facility of the Walter Reed Army Institute of Research at Brooks City-Base, TX. The GTEM cell is approximately $1.8 \mathrm{~m}$ long, $0.9 \mathrm{~m}$ wide, and $0.9 \mathrm{~m}$ high. The septum and top surface, or ceiling, rise at angles of $11^{\circ}$ and $22^{\circ}$, respectively, relative to the bottom surface, or floor. Fig. 1(c) shows the interior of the GTEM cell loaded with four upright T-25 flasks. The flasks are supported in a foam frame on the floor of the GTEM cell at a distance of $72.2 \mathrm{~cm}$ from the source plane shown in Fig. 1(b). The foam frame elevates the flasks $2.5 \mathrm{~cm}$ above the floor of the GTEM cell. The dimensions of the bottom of each flask are $5 \mathrm{~cm}$ by $2.5 \mathrm{~cm}$. The flasks are filled with a cell culture medium to a height of $1 \mathrm{~cm}$.

The FDTD computational domain used to model the GTEM cell is comprised of a uniform Cartesian lattice of cubic grid cells. As shown in Fig. 2 (septum not shown), we oriented the floor of the GTEM cell in the $y-z$ plane of the FDTD grid. We extended the source end of the GTEM cell to create a feed point at the apex where the TEM wave is excited, thereby eliminating the need to model the complex feed structure of the actual GTEM cell. The load end of the GTEM cell was truncated using perfectly matched layer (PML) absorbing boundary conditions [27], which play the same role as the physical absorbers present in the actual GTEM cell in the region beyond the truncation plane. All metal surfaces were treated as perfect electric conductors.

Fig. 3(a) shows two different UWB pulses used as excitation waveforms in this study, along with their corresponding amplitude spectra [Fig. 3(b)]. The solid curve illustrates the following double exponential pulse waveform:

$$
f(t)=A\left(e^{-\alpha t}-e^{-\beta t}\right)
$$

with $\alpha=2.13 \times 10^{9}$ and $\beta=6.74 \times 10^{9}$. Our use of this waveform was motivated by the fact that it provides the closest 


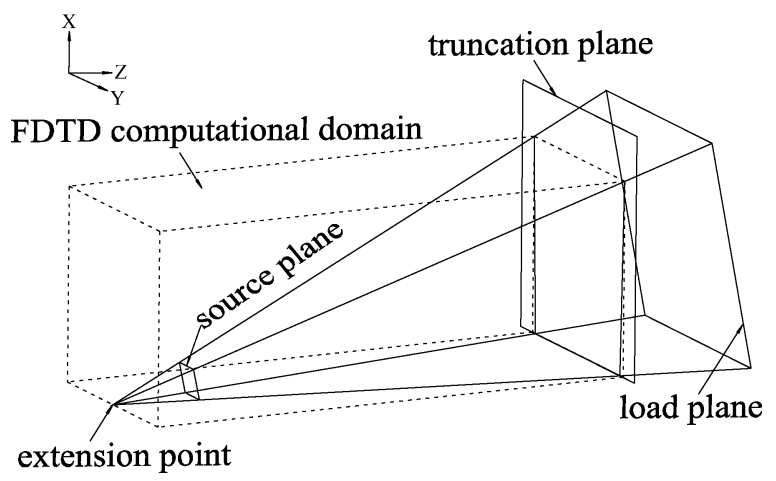

Fig. 2. Three-dimensional schematic showing how the GTEM cell of Fig. 1 was truncated at the load end and extended at the source end to create the FDTD model in a Cartesian grid.

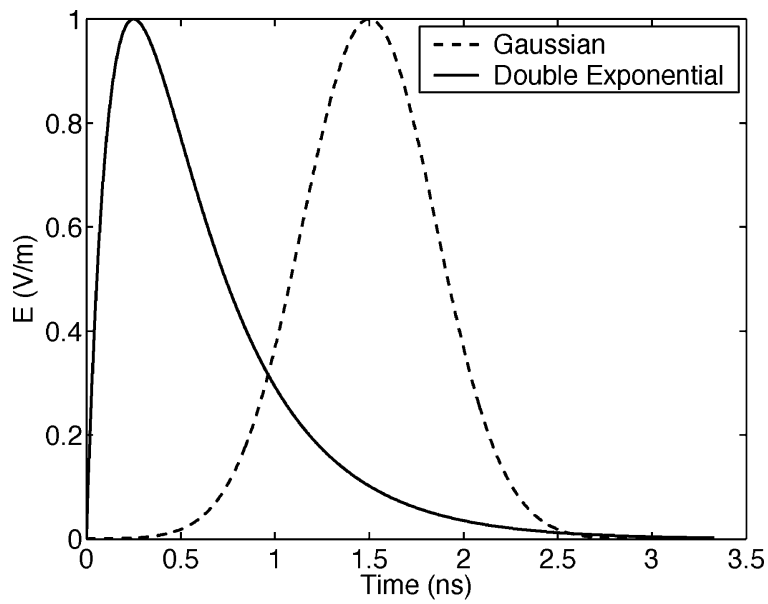

(a)

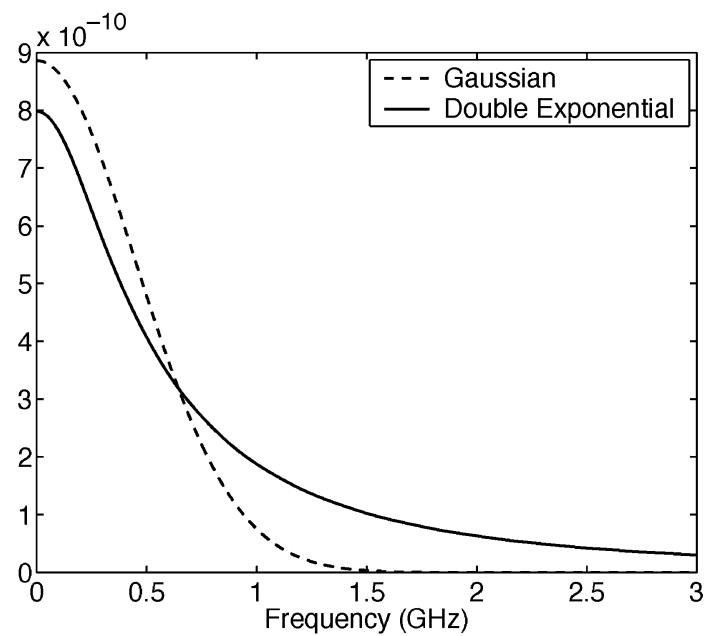

(b)

Fig. 3. (a) Excitation waveforms used in the FDTD simulations: a double exponential pulse and a Gaussian pulse. (b) Amplitude spectrum of each pulse.

functional fit to the types of pulse shapes produced by the experimental hardware. The dotted curve illustrates a Gaussian pulse waveform

$$
f(t)=A e^{-\frac{\left(t-t_{0}\right)^{2}}{\tau^{2}}}
$$

with $\tau=0.5 \times 10^{-9} \mathrm{~s}$ and $t_{0}=3 \tau$. Both waveforms have a pulse width of roughly $1 \mathrm{~ns}$. However, in contrast to the double exponential pulse, the frequency content of the Gaussian pulse is negligible above $1 \mathrm{GHz}-$ a useful feature that will be exploited further in Section IV.

The tapered side walls, septum, and ceiling were resolved to $2.5 \mathrm{~mm}$ using a staircase approximation. Staircasing errors have been found to be negligible when the staircase diagonal is smaller than $\lambda / 2$, where $\lambda$ is the wavelength corresponding to the highest significant frequency in the excitation [28]. With a space increment of $2.5 \mathrm{~mm}$, the largest diagonal in our staircase approximation is on the order of $\lambda_{0} / 20$, where $\lambda_{0}$ is free-space wavelength at $1 \mathrm{GHz}$. Therefore, we conclude that our grid resolution is fine enough to keep artifacts due to the staircase approximation to a minimum, thereby eliminating the need for conformal gridding. We did not exploit the symmetry exhibited by the empty GTEM cell in the $x-z$ plane at the center $y$-coordinate because doing so would preclude simulating asymmetrical flask configurations. The resulting computational domain for the model of the entire GTEM cell contained $280 \times 280 \times 700$ grid cells.

In the FDTD model of the T-25 flasks, we excluded the 1-mm-thick polystyrene flask walls $\left(\epsilon_{r}=2.6, \sigma \simeq 0\right)$. We conducted FDTD simulations of a GTEM cell loaded with a single flask with and without the plastic walls. We determined that the plastic walls have no significant effect on the electric field waveforms and dosimetry calculation inside the flask. Therefore we decided to exclude them in our simulations. This finding is physically consistent with the fact that the walls are electrically thin and have a very low dielectric constant relative to the cell culture medium. Therefore, we modeled each flask as a homogeneous volume $(5 \mathrm{~cm} \times 2.5 \mathrm{~cm} \times 1 \mathrm{~cm})$ comprised only of the cell culture medium.

We modeled the dispersive dielectric properties of the cell culture medium using a Debye model with $\epsilon_{s}=77.43, \epsilon_{\infty}=$ 6.01, $\sigma=1.35 \mathrm{~S} / \mathrm{m}$ and $\tau_{p}=9.41 \mathrm{ps}$. The Debye parameters were adapted from the results of [29] to more closely fit our measured data for the dielectric properties of several commonly used cell culture media for MM6, HAEC, HK293, HL-60, and 244B cells. An auxiliary differential equation technique [22] was used to implement the frequency dependence of the Debye model in our FDTD simulations. We implemented averaging of material properties at the boundaries between the dispersive and nondispersive media in the grid following the approach of [30].

We applied a $z$-directed voltage source across a single grid cell between the septum and the outer wall to excite the UWB pulse at the apex of the modeled GTEM cell. Two types of data were extracted from each FDTD simulation. First, we recorded temporal variations of the three electric field vector components observed at specific observation points in the grid. The observation point used in simulations of the empty GTEM cell is shown in Fig. 4(a). Observation points used in simulations of the loaded GTEM cell are illustrated in Fig. 4(b). We note that each flask was positioned in the GTEM cell so that field observation point FP-2 of Fig. 4(b) lies in the observation plane of 


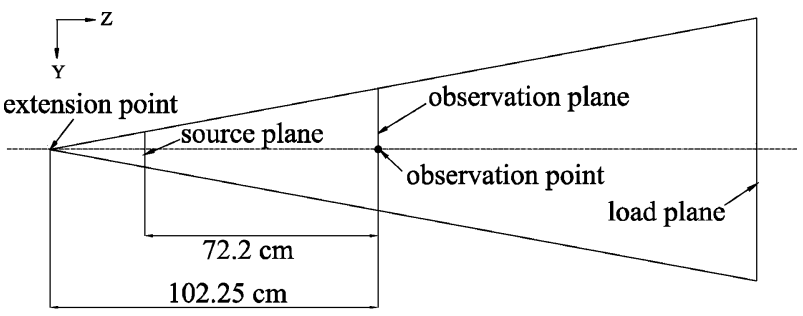

(a)

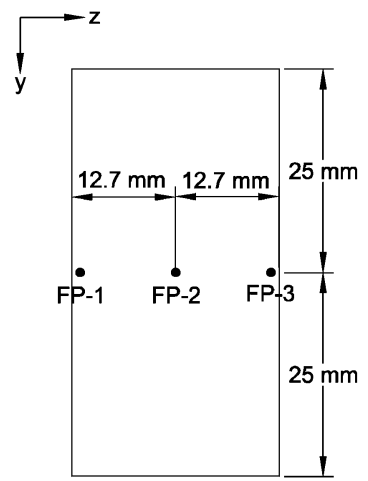

(b)

Fig. 4. (a) Schematic showing the location of the field observation point on the floor of the GTEM cell. (b) Schematic showing the locations of field observation points inside a flask near its bottom surface.

Fig. 4(a). Second, we calculated the spatial distribution of the AED, which for the Debye medium is calculated as

$$
\begin{aligned}
w_{a b s}(x, y, z)= & \int\left[\sigma(x, y, z)|\vec{E}(x, y, z, t)|^{2}\right. \\
& \left.+\vec{J}_{p}(x, y, z, t) \cdot \vec{E}(x, y, z, t)\right] d t,\left[\frac{J}{m^{3}}\right]
\end{aligned}
$$

where $\overrightarrow{J_{p}}$ is the polarization current associated with the Debye pole, as defined in [22]. Since the vector field components are staggered in the FDTD grid, we employed spatial averaging to obtain the three vector components of the electric field and polarization current at common $(x, y, z)$ coordinates of interest in the grid.

\section{VALIDATIONS OF THE FDTD MODEL}

\section{A. Comparison of FDTD and Experimental Characterizations of the Empty GTEM Cell}

First, we conducted an FDTD simulation of UWB pulse propagation in the empty GTEM cell and recorded the temporal variations in the electric and magnetic fields at the observation point shown in Fig. 4(a) at a distance of $2.5 \mathrm{~mm}$ above the floor of the GTEM cell. The double-exponential pulse of Fig. 3(a) and (b) was used as the source excitation waveform in this simulation. Near the floor of the GTEM cell, the dominant electric field vector component in the propagating TEM wave is $E_{x}$. The solid curve in Fig. 5 shows this vertical electric field recorded as a function of time at the specified observation point. We verified that the very low level oscillation present on the tail of

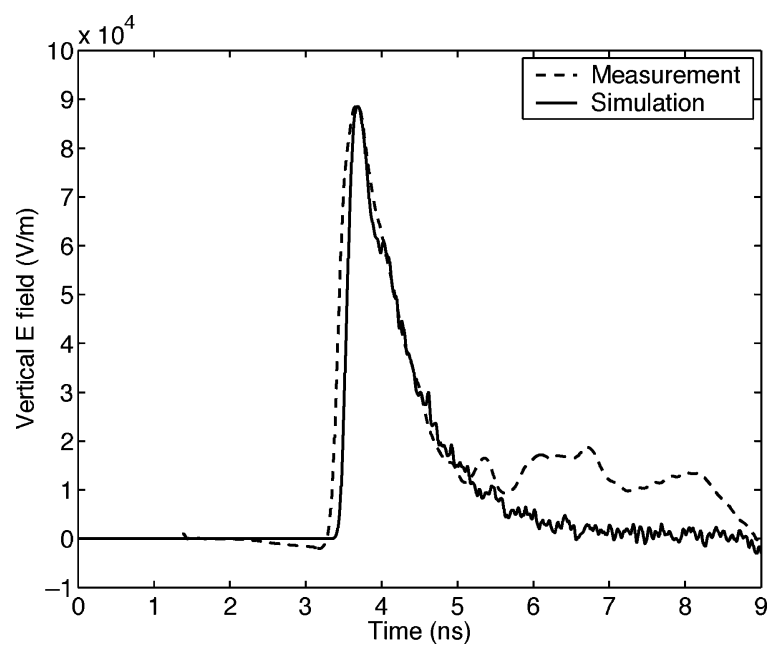

Fig. 5. Comparison of the simulated and measured vertical electric field $\left(E_{x}\right)$ at the observation point of Fig. 4(b) in the empty GTEM cell. Here, the double exponential pulse was used as the excitation waveform in the FDTD simulation.

the FDTD waveform can be reduced to negligible levels by increasing the grid resolution. However, this artifact, which is attributed to the staircase approximations employed in modeling the flared surfaces of the GTEM cell, has no noticeable effect on the dosimetry calculations and therefore further grid refinement was deemed unnecessary. We calculated the wave impedance using the FDTD-computed electric and magnetic field intensities at the point in time when the peak of the pulse passes the observation point. The ratio of the electric and magnetic field intensities was found to be approximately $377 \Omega$. This calculation confirmed that the FDTD model of the GTEM cell properly simulated the propagation of a TEM wave.

For comparison, we also considered the actual waveform measured in the empty GTEM cell with a D-Dot sensor placed at the same observation point as that assumed in the FDTD simulation. The D-dot sensor was an EG\&G grounded asymmetrical conical dipole [31]. The measured waveform, proportional to $d D / d t$, was corrected for cable loss, the limited bandwidth of the measuring equipment, and distortions introduced by the probe itself. Then, it was converted into $E(t)$, as shown by the dotted curve in Fig. 5.

We observe excellent agreement in the overall characteristics of the measured and simulated UWB pulse waveforms in the empty GTEM cell up until $5 \mathrm{~ns}$ at which time the measured waveform exhibits an anomalous, low-amplitude, slowtimescale feature. The late time artifact in the measured waveform may be attributed to impedance mismatches in the GTEM cell and imperfections in the sensor. We verified that this latetime artifact, which appears after the primary pulse, does not contribute to the coupling of the electromagnetic wave into the medium flask, as discussed in Sections IV and V.

\section{B. Effect of the Flask Configuration}

Second, we conducted FDTD simulations to determine the flask configuration to be used in the remainder of the study. Specifically, we explored the extent to which the dosimetry results depended on the number of flasks assumed in the model of the GTEM cell as well as their positions. We considered three 
flask configurations. In the first scenario, we oriented the four flasks as shown in Fig. 1(c). In the second scenario, three of the four flasks were removed from the model leaving only an outmost flask ("flask 1"). In the third scenario, the single flask was re-positioned so that it was centered with respect to the $y$-direction. All simulations were conducted using the double exponential pulse as the source excitation waveform.

The FDTD-computed vertical electric field component, $E_{x}$, was recorded as a function of time at observation point FP-1 in each flask. The similarity of the waveforms observed in the offset flask (flask 1), with and without the other three flasks present, suggests that there are essentially no electromagnetic interactions between the flasks. Furthermore, a comparison of either offset-flask (second scenario) waveform with the waveform observed in the single central flask (third scenario) reveals that the strongest field intensities inside the flask occur when the flask is positioned along the central axis. The AED distributions were also computed in a horizontal plane located $2.5 \mathrm{~mm}$ above the bottom surface of the flask. The general nonuniformity observed in the AED is discussed more extensively in Section V. Here, we simply note that the greatest nonuniformity and the largest AED values were obtained for the case of third scenario. Therefore we conclude that the worst case scenario in terms of field nonuniformity and variation between maximum and minimum AED in the cell culture medium corresponds to the scenario where the GTEM cell is loaded with a single flask positioned along the central axis. This is the flask configuration we choose to examine in the remainder of this paper.

\section{INVESTIGATION OF WAVEFORM REVERBERATION EFFECTS InSide the Cell Culture Medium}

While conducting the validation simulations, we observed that when the double exponential pulse waveform propagated in the empty GTEM cell, the waveform remained monopolar and relatively smooth. In contrast, when the double exponential pulse waveform propagated in the GTEM cell loaded with flasks containing cell media, the electric field waveform (sampled inside the dielectric media) acquired a significant and persistent oscillation. This conversion of a smooth monopulse waveform into a fluctuating waveform could have significant implications for the UWB electromagnetic pulse field interactions with biological cells in an experiment. For example, prior experimental studies [32], [33] indicate that persistent, narrowband high-frequency oscillating electric fields are less effective at electroporating cell membranes than are transient, intense, monopolar electric fields. Therefore, it was important to understand why the initially monopolar pulses acquired the persistent oscillations.

Animated space-time visualizations (not included in this paper) of the electric fields inside and outside the simulated flask motivated a hypothetical explanation for the persistent oscillations. The hypothesis was that the rectangular volume of simulated cell culture medium was acting like a dielectric resonator with a high permittivity. It was speculated that the double exponential pulse was illuminating this dielectric resonator volume with an ultra-broadband of frequency components, one or more of which stimulated resonant eigenmodes. The persistent oscillations of the electric field, well after the

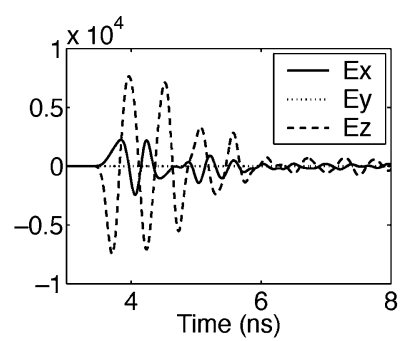

(a)

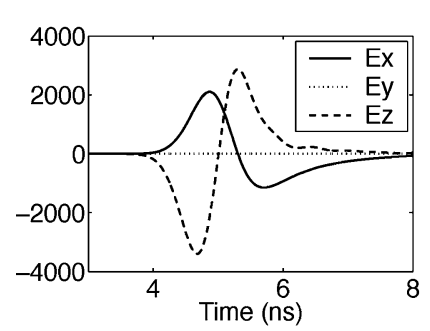

(c)

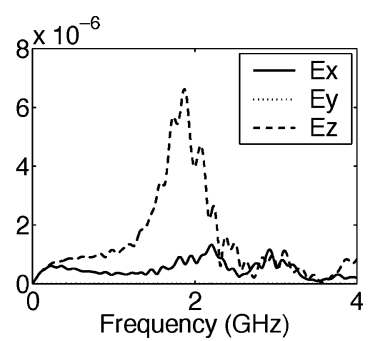

(b)

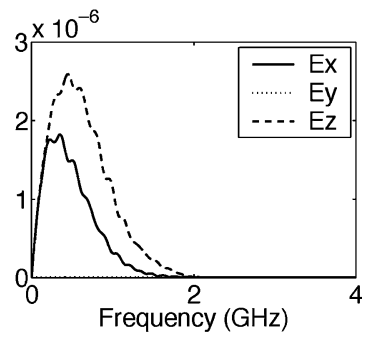

(d)
Fig. 6. Electric fields and their spectra inside the single central flask. (a) Waveforms and (b) spectra of the electric field response for the double exponential pulse. (c) Waveforms and (d) spectra of the electric field response for the Gaussian pulse.

pulse should have passed by the flasks, would represent the field reverberations or a "ringing down" of these moderately damped resonant cavity modes.

Approximate calculations of the resonant eigenfrequencies of a rectangular volume of the cell culture medium were made by modeling the rectangular volume as a dielectric-filled cavity with perfect magnetic conductor (PMC) boundaries. Using the dimensions of the cell culture medium volume and the experimentally measured complex permittivity values, we estimated the lowest order modes of such a rectangular cavity to be $f_{011}^{T M} \simeq 0.75 \mathrm{GHz}, f_{101}^{T E} \simeq 1.84 \mathrm{GHz}, f_{110}^{T M} \simeq 1.74 \mathrm{GHz}$, $f_{111}^{T M, T E} \simeq 1.87 \mathrm{GHz}, f_{021}^{T M} \simeq 0.92 \mathrm{GHz}, f_{120}^{T M} \simeq 1.82 \mathrm{GHz}$, and $f_{121}^{T M, T E} \simeq 1.94 \mathrm{GHz}$. Comparing these values to the frequency spectrum in Fig. 3(b), it is clear that the double exponential pulse has significant spectral content spanning these frequencies.

Fig. 6(a) shows the waveforms of the three vector components of the electric field inside the flask after excitation by the 1-ns double exponential pulse. All three waveforms exhibit persistent reverberations, with the $z$-component's amplitude dominating the other two. Fig. 6(b) shows the frequency spectra of these three waveforms. Comparing these spectra to the incident pulse spectra of Fig. 3(b), it is evident that the electric field waveforms inside the flask are dominated by spectral content near $2.0 \mathrm{GHz}$ while the spectral content of the incident pulse below $1.5 \mathrm{GHz}$ has been lost or dissipated. This is a strong indication that the 1-ns double exponential incident pulse has stimulated one or more of those dielectric cavity resonances near $2.0 \mathrm{GHz}$. In particular, it is speculated that the $\mathrm{TM}_{111}$ mode is excited. This is discussed later in the paper.

A second test of the hypothesis was conducted by exciting the GTEM cell with the 1-ns Gaussian pulse of Fig. 3(a). This was motivated by the observation that the 1-ns Gaussian pulse would contain negligible spectral content near $2 \mathrm{GHz}$, where 
the resonances of the cell culture medium appear to predominate. Fig. 6(c) and (d) displays the results. In Fig. 6(c), we observe none of the oscillatory reverberations that were observed in Fig. 6(a) for the double exponential pulse. In Fig. 6(d), we observe that the spectral content of the electric field waveforms recorded inside the medium do not display any evidence of "filtered" resonances near $2 \mathrm{GHz}$. The Gaussian pulse did not stimulate such reverberations because it lacked significant spectral content near $2 \mathrm{GHz}$. This frequency regime apparently corresponds to the eigenfrequencies of the resonant modes most likely to be stimulated by the particular polarization of the fields of these incident pulses.

We also conducted a simulation using a replica of the measured waveform (dashed line in Fig. 5) as the source excitation waveform and found that the resulting electric waveforms inside the cell culture medium were almost identical to the case of using the double exponential pulse as the source excitation. This clearly shows that only the short-time-scale features couple into the interior of the cell culture medium volume: as claimed in Section III-A, the slow-timescale tail feature of the measured waveform is ignorable. These results are completely consistent with the observations in [18] where only the fast-rise-time leading edge portion of their pulse penetrated the interior of the sample, while the slowly decaying trailing edge did not couple.

The final evidence confirming that the incident 1-ns double exponential pulse was coupling to resonant modes in the cell culture medium volume, leading to the reverberations, comes from the observation in Fig. 6(a). After excitation, the dominant field component of the stimulated resonances was the $z$-component even though the incident pulse's electric field was dominated by an $x$-polarization. We believe that this is a part of a holistic, self-consistent understanding of the electromagnetic interaction between the incident UWB pulses and the mediumcontaining flasks. This will be discussed after the introduction and discussion of some additional interesting observations.

\section{Dosimetry CAlCulations}

As outlined in the Introduction, the objective of the UWB exposure experiments is to conduct investigations of nonthermal effects in biological cells due to UWB EMP exposure. Accurate interpretation of the experimental data requires an accurate knowledge about whether all cells were exposed to the same electric field conditions, or whether significant field nonuniformities were present within the cell culture medium contained in the flasks. For the latter case, a knowledge of the percentage of cells in different AED exposure ranges becomes important. In addition, in order to confirm that any cellular or subcellular effects observed in these experiments resulted from nonthermal mechanisms, it is important to confirm that the cells were exposed to a negligible temperature rise during the exposures. With these issues in mind, we present and discuss the results of FDTD dosimetry calculations for the GTEM cell loaded with a single flask that is partially filled with cell culture medium.

AED distributions were calculated for three horizontal layers (vertically positioned near the bottom, the middle, and the top of the cell medium volume). The peak electric field of the incident pulse was about $100 \mathrm{kV} / \mathrm{m}$ (Fig. 5). The results are plotted in Fig. 7(a)-(f), where the lower layer is $2.5 \mathrm{~mm}$ above the bottom

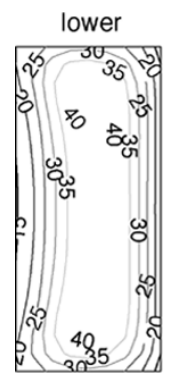

(a)

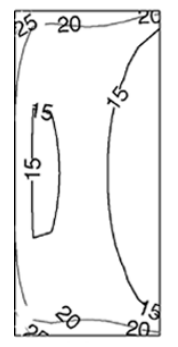

(d)

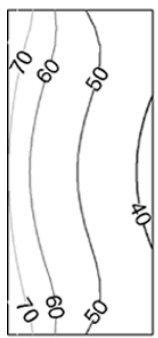

(g)

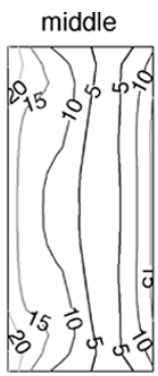

(b)

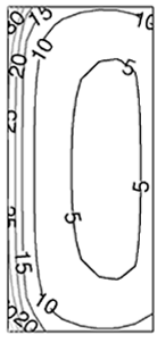

(e)

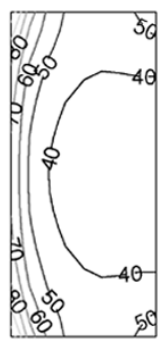

(h)

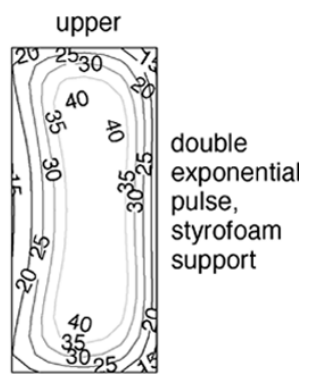

(c)

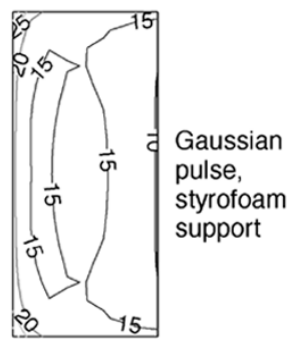

(f)

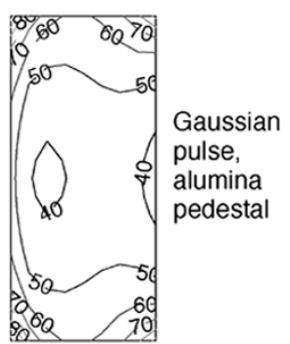

(i)
Fig. 7. Absorbed energy density $\left(\mathrm{mJ} / \mathrm{m}^{3}\right)$ distributions in the lower, middle and upper layers of the flask for the double exponential pulse with the flask supported by a foam frame [(a)-(c)], the Gaussian pulse with the flask supported by a foam frame $[(d)-(f)]$ and the Gaussian pulse with the flask supported by an alumina pedestal $[(\mathrm{g})-(\mathrm{i})]$.

of the medium, the middle layer is $2.5 \mathrm{~mm}$ above the lower layer, and the upper layer is $2.5 \mathrm{~mm}$ above the middle layer, and $2.5 \mathrm{~mm}$ below the top of the cell medium volume.

The first observation from Fig. 7(a)-(f) is that the AED distributions inside the cell culture medium are highly nonuniform, both vertically and horizontally, for both the double exponential and the Gaussian pulse excitations. In both types of excitations, the AED in the middle layer was uniformly extremely low, compared to AED levels in the upper and lower layers. For example, in Fig. 7(a) and (c), the AED reaches approximately $50 \mathrm{~mJ} / \mathrm{m}^{3}$ in the centers of the upper and lower layers, but is only about $5 \mathrm{~mJ} / \mathrm{m}^{3}$ in the center of the midplane in Fig. 7(b). Similarly, for the Gaussian pulse excitation, the AED exceeds $15 \mathrm{~mJ} / \mathrm{m}^{3}$ in the upper and lower sampling planes [Fig. 7(d) and (f)], but is less than approximately $5 \mathrm{~mJ} / \mathrm{m}^{3}$ in the midplane of Fig. 7(e).

After studying animated space-time visualizations of the electric fields associated with the EMP incident on the cell culture medium volume, we constructed a hypothesis to explain the nonuniformities. As illustrated in Fig. 8, as the electromagnetic pulse propagates along the $z$-dimension and encounters the high-dielectric-constant cell culture medium volume, it experiences refraction. The energy velocity in the cell culture 


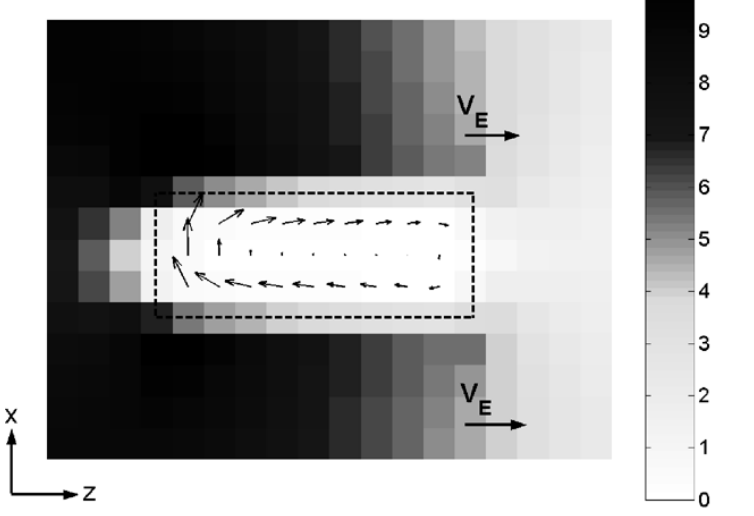

(a)

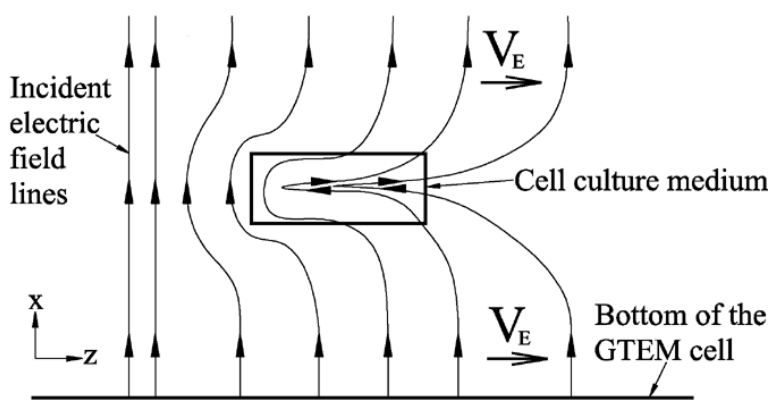

(b)

Fig. 8. Electric field phenomena in a cross-sectional $(x-z)$ plane through the cell culture medium. (a) Vector plot inside the cell culture medium and total electric field intensity plot outside the cell culture medium. (b) Didactic drawing of electric field lines inside and outside the cell culture medium. $V_{E}$ is the energy velocity outside the cell culture medium.

medium is dramatically slower than the energy velocity in free space. As revealed in Fig. 8(a) and illustrated schematically in Fig. 8(b), this leads to a distortion of the EMP wavefronts and a refractive bending of the electric field vectors outside and surrounding the cell culture medium volume. As the E-field vectors are refracted above and below the medium volume from initially $x$-polarized to eventually $z$-polarized, they become oriented tangential to the medium surface. In this orientation they are able to most effectively couple into the medium, consistent with continuity conditions for tangential components of electric fields. Fig. 8 also explains the reason why the AED of the vertical midplane region is nearly uniformly zero, for both types of pulse excitations [see Fig. 7(b) and (e)]. As illustrated in Fig. 8, the refraction of the EMP outside the cell culture medium results in a rotation of the electric field vectors above and below the volume from primarily $x$-polarized to primarily $z$-polarized. Therefore, the EMP's electric fields propagate from the free space exterior into the medium interior with a polarization predominantly in the $z$ or axial direction. As the energy penetrates from both above and below, the electric fields reach the midplane where they experience near-total cancellation due to opposing field orientations, as illustrated in Fig. 8(b). This produces a null in the electric field distribution along the midplane of the cell medium volume, and similarly results in a null in the AED along this midplane.
The above insights regarding the refraction of the electric field, as it encounters the cell medium volume, explain the previously reported observation that the dominant electric field component observed inside the cell medium was a $z$-component, even though the incident pulse's electric field was primarily $x$-polarized (see Fig. 6(a), for example).

Comparing Fig. 7(a)-(d) and (c)-(f), we observe that the AED is much more nonuniform in the upper and lower horizontal planes for the double exponential pulse excitation than for the Gaussian pulse excitation. This is explained by the fact that the double exponential pulse excited nonuniform resonant cavity eigenmodes in the cell culture medium while the Gaussian pulse did not excite such modes. As discussed previously, there are approximately 7 eigenmodes with resonant frequencies approximately between 1.7 and $2.0 \mathrm{GHz}$. However, only the $\mathrm{TM}_{111}^{z}$ mode has field orientations that are consistent with the field polarities of the incident field external to the cell culture medium after refraction. The presence of the dominant $E_{z}$ field components rule out the possibility that the $\mathrm{TE}_{111}^{z}$ or $\mathrm{TE}_{121}^{z}$ modes have been excited. Moreover, the $\mathrm{TM}_{121}^{z}$ and $\mathrm{TM}_{120}^{z}$ modes were not likely stimulated because the incident external electric field is symmetrical across the cell culture medium in the $y$-direction and would therefore only excite odd-number modes in that direction.

The nonuniform AEDs of Fig. 7(a)-(f) are generally not desirable in biological cell exposure experiments. In particular, recognizing that the weak $A E D$ in the midplane is due to field cancelation resulting anti-symmetrical field refraction above and below the cell medium volume (see Fig. 8), an apparent fix would be to replace the styrofoam support with a high-dielectric-constant material pedestal underneath the flask. The effect of this would be to reduce the refraction of the electromagnetic fields underneath the flask, disrupting the above-below antisymmetry. Ideally, the electric field cancelation observed in the midplane of the medium [Fig. 7(b) and (e)] would be eliminated and we should expect an increase of the electric field inside the volume of the cell culture medium.

A new simulation model was created in which the foam underneath the flask was replaced by alumina $\left(\epsilon_{r}=9.6\right)$. The concept was tested using the Gaussian pulse excitation. The AED from this configuration is compared to that obtained with a foam support in Fig. 7(d)-(i). The uniformity between the three horizontal sampling layers has clearly been improved, with a dramatic increase in the AED in the middle layer [compare Fig. 7(e) and (h)]. Moreover, the overall AED has been dramatically increased in all three layers compared to the results obtained with the foam.

With the computed AED results, we also estimated the maximum temperature rises that one might expect from these types of exposures. To place an upper bound on the temperature transients and to simplify the calculations, conductive and convective heat transfer were assumed to be negligible during the short, nanosecond exposure times. Each $2.5 \mathrm{~mm} \times 2.5 \mathrm{~mm} \times 2.5 \mathrm{~mm}$ simulation voxel of the cell culture medium was treated as an independent thermal volume using the heat capacity $(4186 \mathrm{~J} / \mathrm{KgK})$ and mass density $\left(1000 \mathrm{Kg} / \mathrm{m}^{3}\right)$. The estimated single pulse maximum temperature rise was on the order of $10^{-8} \mathrm{~K}$. In the multi-pulse UWB exposure experiments conducted by the 
University of Texas Health Science Center, the pulse repetition rate was 250 pulses per second and the total duration was 30 minutes. Under these exposure conditions, we conclude that a conservative upper bound for the maximum possible temperature rise due to the pulse train is on the order of $10^{-3} \mathrm{~K}$. This verifies that the temperature rise is negligible during these UWB EMP exposure experiments, which is consistent with [18]. Consequently, any unusual cell function or histopathology can be associated with nonthermal effects.

\section{InVESTIGATION OF EleCtric Field Polarity}

As mentioned in an earlier section, published experimental studies [32], [33] indicate that narrowband oscillating electric fields or even UWB bipolar electric field pulses interact differently with biological materials than intense monopolar electric field pulses. The intention of the experimental design of Fig. 1 was to expose biological cells to an intense, monopolar electric field waveform such as the waveform of Fig. 5. Having discovered, through simulations, that the electric field waveforms inside the cell culture medium can be dramatically modified by refraction and reverberation effects, we examined more closely the time-dependent electric field polarizations that cells would be exposed to inside the medium contained in the flask. To investigate the time-dependent electric field polarization, the vector components $E_{x}$ and $E_{z}$ (the two dominant components evident in Fig. 6) were recorded at a representative observation point in the medium ( $E_{y}$ is very small and can be neglected). To simplify the problem, the Gaussian pulse excitation which did not stimulate reverberating resonant cavity modes was studied. Illustrative $E_{x}$ and $E_{z}$ waveforms recorded at the observation point are displayed in Fig. 9(a). From these waveforms, eight discrete samples of $E_{x}$ and $E_{z}$ were taken, uniformly spaced in time throughout the pulsed field duration within the dielectric medium. The results are plotted in an $E_{x}-E_{z}$ coordinate vector space [Fig. 9(b)]. It is clearly evident from Fig. 9(b) that although the incident pulse's electric field is linearly polarized and monopolar, the electric field inside the cell culture medium has a rotating polarization and that biological cells suspended in this medium will not see a monopolar electric field. For the double exponential EMP used in the experiments, the effect is even more pronounced, due to the stimulation of persistent, reverberating cavity eigenmodes.

\section{SUMMARY}

GTEM transmission cells have been previously used to experimentally study exposure of biological cells to $\sim 1 \mathrm{~ns}$, UWB, monopolar, electromagnetic pulses having spectral content from dc to the $\mathrm{GHz}$ range of frequencies. Using high resolution, 3-D FDTD simulations, we have investigated the time-dependent electric field waveforms and time-integrated energy dose spatial distributions within a finite volume of biological cell culture medium during these experiments. The simulations agree well with experimental measurements of waveforms in an empty GTEM cell, and predict a highly uniform field distribution that closely approximates a TEM wave. However,

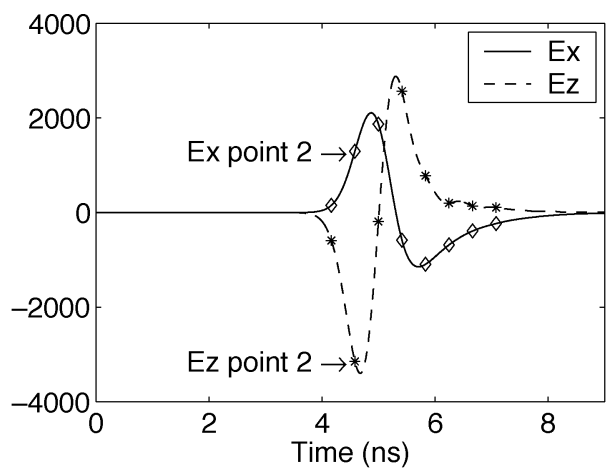

(a)

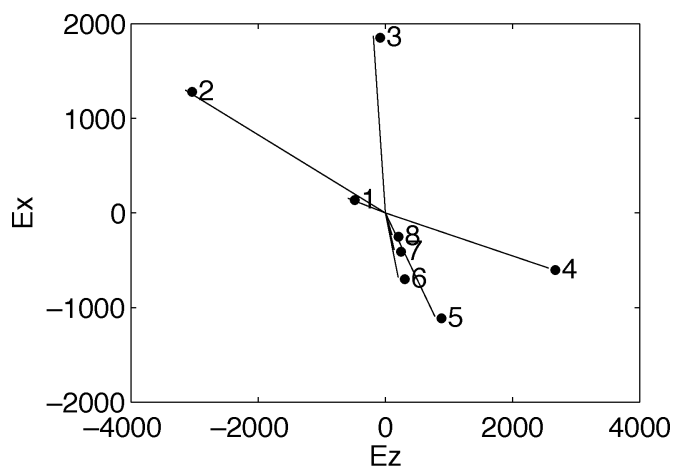

(b)

Fig. 9. Electric field polarization inside the single central flask. (a) Electric field waveforms. (b) Mapped electric fields.

when one or more flasks containing cell culture media are placed inside the GTEM cell, the uniform fields of the empty GTEM cell are significantly perturbed. The fields inside the cell culture medium, representing the fields to which the biological cells are exposed, are no longer monopolar and are spatially highly nonuniform. We have observed these results for several flask orientations, indicating that the result is a fairly general one. This has important implications for experimental design, since it has been shown elsewhere that bipolar wideband, or persistent and oscillatory narrowband electric fields are less effective at inducing effects such as electroporation than are transient, intense, monopolar electric field pulses.

The simulations were able to show that the electromagnetic field oscillation and nonuniformity effects result from a combination of refraction and distortion of the incident wave, combined with excitation of resonant eigenmodes within the cell culture medium volume. It is clear that these electrically small specimen volumes act as high frequency bandpass filters, with strongest coupling having a strong association with dielectric resonator eigenmodes. It was further shown that these distortions of the incident waveform may be mitigated by supporting the sample on a high permittivity pedestal and by modifying the incident waveform to more closely approximate a Gaussian pulse. Under all simulated conditions, the estimated maximum temperature rises are completely negligible, ensuring that any experimentally observed unusual cell function or histopathology can be associated with nonthermal effects. 


\section{REFERENCES}

[1] M. Natarajan, B. K. Nayak, C. Galindo, S. P. Mathur, F. N. Roldan, and M. L. Meltz, "Nuclear translocation and DNA-binding activity of NF-kB upon pulsed ultra-wideband electromagnetic field exposure fails to transactivate kB-dependent gene expression in human monocytes," Radiat. Res., 2006, to be published.

[2] R. L. Seaman, J. E. Parker, J. L. Kiel, S. P. Mathur, T. R. Grubbs, and K. Prol, "Ultra-wideband pulses increase nitric oxide production by RAW 274.7 macrophages incubated in nitrate," Bioelectromagnetics, vol. 23, pp. 83-87, 2002.

[3] S. T. Lu and S. P. Mathur, "Hypotension induced by ultra-wide-band pulses: dose response, replication, orthostatic response, and heart rate variability," in Proc. 2nd Int. Workshop on Biological Effects of EMF's, P. Kostarakis, Ed., 2002, pp. 409-418.

[4] Vijayalaxmi, R. L. Seaman, M. L. Belt, J. M. Doyle, S. P. Mathur, and T. J. Prehode, "Frequency of micronuclei in the blood and bone marrow cells of mice exposed to ultra-wideband electromagnetic radiation," Int. J. Radiat. Biol., vol. 75, no. 1, pp. 115-120, 1999.

[5] R. L. Seaman, M. L. Belt, J. M. Doyle, and S. P. Mathur, "Hyperactivity caused by a nitric oxide synthase inhibitor is countered by ultra-wideband pulses," Bioelectromagnetics, vol. 20, pp. 431-439, 1999.

[6] _ - "Ultra-wideband electromagnetic pulses and morphine-induced changes in nociception and activity in mice," Physiol. Behav., vol. 65, pp. 263-270, 1998.

[7] O. N. Pakhomova, M. L. Belt, S. P. Mathur, J. C. Lee, and Y. Akyel, "Ultra-wide band electromagnetic radiation does not affetc UV-induced recombination and mutagenesis in yeast," Bioelectromagnetics, vol. 19, pp. 128-130, 1998.

[8] J. R. Jauchem, R. L. Seaman, H. M. Lehnert, S. P. Mathur, K. L. Ryan, M. R. Frei, and W. D. Hurt, "Ultra-wideband electromagnetic pulse: lack of effects on heart rate and blood pressure during two-minute exposures of rats," Bioelectromagnetics, vol. 19, pp. 330-333, 1998.

[9] O. N. Pakhomova, M. E. Belt, S. P. Mathur, J. C. Lee, and Y. Akyel, "Lack of genetic effects of ultrawide-band electromagnetic radiation in yeast," Electro-Magnetobiol., vol. 16, pp. 195-201, 1997.

[10] C. J. Sherry, D. W. Blick, T. J. Walters, G. C. Brown, and M. R. Murphy, "Lack of behavioral effects in nonhuman primates after exposure to ultrawideband electromagnetic radiation in the microwave frequency range," Radiat. Res., vol. 143, no. 1, pp. 93-97, 1995.

[11] S. A. Miller, M. E. Bronson, and M. R. Murphy, "Ultrawideband radiation and pentylenetetrazol-induced conclusions in rats," Bioelectromagnetics, vol. 20, pp. 327-329, 1999.

[12] D. Konigstein and D. Hansen, "A new family of TEM-cells with enlarged bandwidth and optimized working volume," in Proc. 7th Int. Zurich Symp. and Tech. Exhibit on EMC, 1987, pp. 127-132.

[13] M. L. Crawford, "Generation of standard EM fields using TEM transmission cells," IEEE Trans. Electromagn. Compat., vol. EMC-16, no. 4, pp. 189-195, Nov. 1974.

[14] K. Huang and Y. Liu, "A simple method for calculating electric and magnetic fields in GTEM cell," IEEE Trans. Electromagn. Compat., vol. 36 , no. 4, pp. 355-358, Nov. 1994.

[15] R. De Leo, T. Rozzi, C. Svara, and L. Zapelli, "Rigorous analysis of the GTEM cell," IEEE Trans. Microw. Theory Tech., vol. 39, no. 3, pp. 488-500, Mar. 1991.

[16] T. E. Harrington, "GTEM fields FDTD modeling," in Proc. IEEE Int. Symp. Electromagnetic Compatibility, 1997, pp. 614-619.

[17] W. A. Radasky, K. S. Smith, D. Hansen, and D. Ristau, "Calculation and measurements of fast EM pulses in the GTEM cell," in Proc. IEEE Int. Symp. Electromagnetic Compatibility, 1996, pp. 52-57.

[18] N. Simicevic and D. T. Haynie, "FDTD simulation of exposure of biological material to electromagnetic nanopulses," Phys. Med. Biol., vol. 50, pp. 347-360, Jan. 2005.

[19] S. Ishigami, K. Harima, and Y. Yamanaka, "Estimation of E-field distribution in a loaded GTEM cell," in Proc. IEEE Int. Symp. Electromagnetic Compatibility, 2001, pp. 129-134.

[20] C. E. Baum, "Electromagnetic sensors and measurement techniques," in Fast Electrical and Optical Measurements, J. E. Thompson and L. H. Leussen, Eds. Dordrecht, The Netherlands: Martinus Nijhoff (Kluwer), 1986.

[21] M. Kanda, "Time-domain sensors and radiators," in Time-Domain Measurements in Electromagnetics, E. K. Miller, Ed. New York: Van Nostrand Reinhold, 1986.

[22] A. Taflove and S. C. Hagness, Computational Electrodynamics: The Finite-Difference Time-Domain Method, 3rd ed. Norwood, MA: Artech House, 2005.
[23] M. Burkhardt, K. Pokovic, M. Gnos, T. Schmid, and N. Kuster, "Numerical and experimental dosimetry of Petri dish exposure setups," Bioelectromagnetics, vol. 17, pp. 483-493, 1996.

[24] M. Popovic, S. C. Hagness, and A. Taflove, "Finite-difference timedomain analysis of a complete transverse electromagnetic cell loaded with liquid biological media in culture dishes," IEEE Trans. Biomed. Eng., vol. 45, pp. 1067-1076, Aug. 1998.

[25] W. F. Pickard, W. L. Straube, and E. G. Moros, "Experimental and numerical determination of SAR distributions within culture flasks in a dielectric loaded radial transmission line," IEEE Trans. Biomed. Eng., vol. 47, pp. 202-208, Feb. 2000.

[26] L. Laval, P. Leveque, and B. Jecko, "A new in vitro exposure device for the mobile frequency of $900 \mathrm{MHz}$, , Bioelectromagnetics, vol. 21, pp. 255-263, May 2000

[27] J. P. Berenger, "A perfectly matched layer for the absorbtion of electromagnetic waves," J. Computational Phys., vol. 114, pp. 185-200, 1994.

[28] J. B. Schneider and K. L. Shlager, "FDTD simulations of TEM horns and the implications for staircased representations," IEEE Trans. Antennas Propagat., vol. 45, pp. 1830-1938, Dec. 1997.

[29] J. Hilland, "Simple sensor system for measuring the dielectric properties of saline solution," Meas. Sci. Technol., vol. 8, pp. 901-910, 1997.

[30] D. Popovic and M. Okoniewski, "Effective permittivity at the interface of dispersive dielectric in FDTD," IEEE Microw. Wireless Components Lett., vol. 13, pp. 265-267, Jul. 2003.

[31] J. Bao, "Picosencond domain electromagnetic pulse measurements in an exposure facility: an error compensation routine using deconvolution techniques," Rev. Sci. Instrum., vol. 68, no. 5, 1997.

[32] J. R. Beveridge, S. J. MacGregor, L. Marsili, J. G. Anderson, N. J. Rowan, and O. Farish, "Comparison of the effectiveness of biphase and monophase rectangular pulses for the inactivation of micro-organizms using pulsed electric fields," IEEE Trans. Plasma Sci., vol. 30, pp. 1525-1531, Aug. 2002.

[33] D. W. Jordan, R. M. Gilgenbach, M. D. Uhler, L. H. Gates, and Y. Y. Lau, "Effects of pulsed, high-power radiofrequency radiation on electroporation of mammalian cells," IEEE Trans. Plasma Sci., vol. 32, pp. 1573-1578, Aug. 2004.

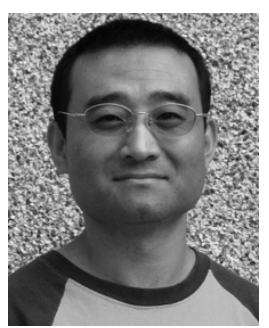

Zhen Ji (S'02) received the B.S. degree from Tsinghua University, Beijing, China, in 1994 and the M.S. degree from Rose-Hulman Institute of Technology, Terre Haute, IN. in 2001, both in electrical and computer engineering. He is currently pursuing the $\mathrm{Ph} . \mathrm{D}$. degree in electrical and computer engineering at the University of Wisconsin-Madison.

His current research interests include biological applications of computational electromagnetics and pulsed electric fields induced biological cell membrane dynamics.

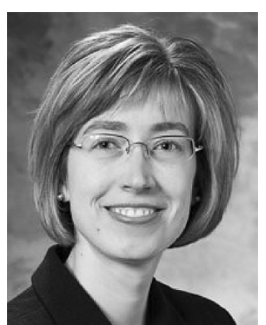

Susan C. Hagness (S'91-M'98-SM'04) received the B.S. degree with highest honors and the Ph.D. degree in electrical engineering from Northwestern University, Evanston, IL in 1993 and 1998, respectively. While working toward the Ph.D. degree, she was a National Science Foundation (NSF) Graduate Fellow and a Tau Beta Pi Spencer Fellow.

Since August 1998, she has been with the University of Wisconsin-Madison, where she is currently an Associate Professor in the Department of Electrical and Computer Engineering and a faculty affiliate of the Department of Biomedical Engineering. Her research interests include FDTD theory and applications in photonics and bioelectromagnetics as well as microwave imaging, sensing, and thermal therapy techniques in biological and medical applications. She co-authored Computational Electrodynamics: The Finite-Difference Time-Domain Method, 3rd. ed. (Artech House, 2005) with A. Taflove.

Dr. Hagness is an elected member of the IEEE Antennas and Propagation Society (AP-S) Administrative Committee and member-at-large of the United States National Committee (USNC) of the International Union of Radio Science (URSI). She is currently an associate editor for the IEEE ANTENNAS AND Wireless Propagation LetTers. She was the recipient of the Presidential Early Career Award for Scientists and Engineers presented by the White House in 2000. In 2002, she received the Booker Fellowship Award from the USNC/ 
URSI. She was also named one of the 100 top young innovators in science and engineering in the world by the Massachusetts Institute of Technology (MIT) Technology Review magazine. In 2003, she received the University of Wisconsin Emil Steiger Distinguished Teaching Award. She received the IEEE Engineering in Medicine and Biology Society Early Career Achievement Award in 2004 and the URSI Isaac Koga Gold Medal in 2005.

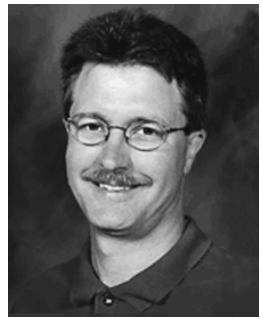

John H. Booske (S'82-M'85-SM'93) received the $\mathrm{Ph} . \mathrm{D}$. degree in nuclear engineering from the University of Michigan at Ann Arbor, in 1985.

From 1985 to 1989 , he was a Research Scientist with the University of Maryland at College Park. In 1990, he joined the faculty of the University of Wisconsin-Madison (UW), where is currently a Professor of electrical and computer engineering. From 2001-2005, he served as Director of the UW Materials Science Program. His research interests include the experimental and theoretical study of coherent electromagnetic radiation sources and their applications with emphasis in the RF, microwave, millimeter-wave, and terahertz regimes. His recent research activities include vacuum electronics, microwave and RF heating of materials, microfabricated $\mathrm{THz}$ waveguide components, biological/biomedical application of microwave and RF fields, and nanoscale transport of dopants in semiconductors. He co-edited Microwave and Radio Frequency Applications (Amer. Ceramics Soc., 2003), and Microwave and Millimeter-Wave Power Electronics (IEEE Press, 2004).

Prof. Booske was a Guest Editor of the IEEE TRAnSACtions on Plasma SCIENCE. He served as co-director of a U.S. Department of Defense (DoD) MURI99 Consortium on Innovative Microwave Vacuum Electronics and as director of a MURI04 consortium on cathode field emission and RF window breakdown in high-power microwave sources. He was the recipient of the University of Wisconsin Vilas Associate Award for research and the U.S. National Science Foundation (NSF) Presidential Young Investigator Award. He was also the recipient of numerous teaching awards including the UW Chancellor's Distinguished Teaching Award and the Benjamin Smith Reynolds Award for Excellence in Teaching Engineering.

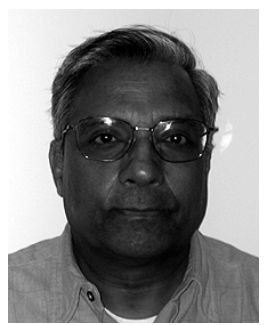

Satnam Mathur (M'80) received the Diploma in electronics and radio engineering (DERE) with highest honors from C. Wadia Institute of Technology, Pune, India, the MS degree in electrical engineering from the University of Windsor, Windsor, ON, Canada, in 1970, and the Ph.D. degree in electrical engineering from Michigan State University, East Lansing, in 1974. He received the MBA degree in Computer Information Science from the University of Phoenix, Phoenix, AZ, in 2004.

He worked as Assistant Professor and Professor of Electrical Engineering at the University of Delhi, Delhi, India, from 1975 to
1987. He was a Research Fellow at the University of Utah, Salt Lake City, from 1987 to 1989 , where he worked on developing FDTD code for modeling the interaction of electromagnetic fields with complex targets and RCS calculations. From 1989 to 2004, he worked as Senior Electrical Engineer at McKesson BioServices, US AMRD, Brooks City-Base, San Antonio, TX, in RF bioeffects research. His main research interests are dosimetry methodology, including numerical dosimetry using FDTD. He is currently teaching Computer Information Technology courses at Coastal Bend College, Pleasanton, TX.

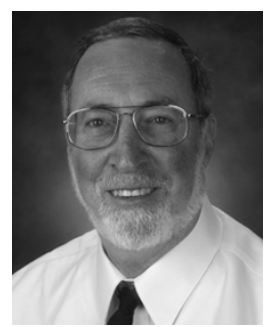

Martin L. Meltz (M'94) received the B.S. degree in physics from the State University of New York at Stony Brook in 1963 and the Ph.D. degree in radiation biophysics from the University of Rochester, Rochester, NY, Jan., 1970.

$\mathrm{He}$ is a Professor with tenure and Chief of the Radiobiology Group in the Department of Radiation Oncology at the University of Texas Health Science Center at San Antonio (UTHSCSA). He was an Atomic Energy Commission Postdoctoral Fellow at the Laboratory of Radiobiology, University of California, San Francisco. He became an Associate Foundation Scientist a the Southwest Foundation for Biomedical Research in San Antonio in 1971, and then joined the faculty at UTHSCSA as an ionizing Radiation Biologist in 1979. He began his studies of the possible genotoxic activity of radio frequency radiation approximately 20 years ago, with funding from the U.S. Air Force. His current research, funded by the U.S. Air Force Office of Scientific Research (AFOSR), is exploring molecular (genomic and proteomic) and cellular events occurring in human cells after exposure to extremely high peak power pulsed electromagnetic fields.

Dr. Meltz is a member of the Bioelectromagnetics Society, the Radiation Research Society, the Environmental Mutagen Society, the Society for Risk Analysis the American Association for the Advancement of Science, and the American Public Health Association. He has served on the Board of Directors of the Bioelectromagnetics Society, and is a past-member of the Committee on Man and Radiation (COMAR) of the Engineering in Biology and Medicine Society. He has more than 62 published articles in refereed scientific journals, 2 published letters, 5 books and book chapters, and 7 technical reports available through the NTIS. He has served as a consultant to industry and as an invited lecturer, focusing on the biological and possible health effects of radiofrequency field exposures. He recently completed voluntary service on the editorial committee of SC-4 of the International Committee on Electromagnetic Safety of IEEE. 